\title{
ON THE INTEGRALS OF A SINGULAR REAL ANALYTIC DIFFERENTIAL FORM IN $R^{n}$
}

\author{
A. MEZIANI
}

\begin{abstract}
In this paper, we study the constancy on the fibers for the continuous integrals of a complex-valued real analytic differential form in $R^{n}$. Then we prove an isomorphism result between the space of smooth integrals and a space built from spaces of Whitney functions.
\end{abstract}

\section{INTRODUCTION}

In [1] and [2], M. S. Baouendi and F. Treves have introduced hypoanalytic structures and studied the functions annihilated by systems of vector fields. Among their results is the constancy on the fibers. More precisely, in codimension 1 , let $Z(x)$ be the germ at $0 \in R^{n}$ of a complex-valued $C^{k}$-function such that $d Z(0) \neq 0$. Then every continuous solution $f$ of $L f=0$, where $L$ is any vector field orthogonal to $d Z$, satisfies the following:

$$
f\left(Z^{-1}(Z(x))\right)=f(x) \text {. }
$$

Here, we consider a germ at $0 \in R^{n}$ of a singular, complex-valued, real analytic differential form $\omega$ and seek an analogous property for its integrals. For this purpose, we start in $\S 1$ by recalling some definitions and some results in the holomorphic category. Then we introduce a fundamental integral $Z$ of $\omega$ (an analogue of $Z$ such that $d Z(0) \neq 0$ in the hypoanalytic theory), define the fiber $F_{x}$ of $\omega$ above the point $x$ as the preimage via $Z$ of $Z(x)$, and write a neighborhood $U$ of 0 as

$$
U=\bigcup_{j=1}^{k} U_{j} \cup F_{0},
$$

where $U_{j}$ 's are the connected components of $U-F_{0}$. Note that real-valued singular differential forms have been the focus of numerous authors (for more details, see Moussu [6]).

$\S 2$ is devoted to the constancy on the fibers of the continuous integrals of $\omega$. Theorem 2.11 says that if $f$ is a continuous integral of $\omega$ in $U$, then $f$ is constant on the fibers in each $U_{j}$ separately. As a consequence of this result, we obtain the constancy on the fibers in $U$ (Theorem 2.12) if the codimension of $F_{0}$ is $\geq 2$.

Received by the editors November 1, 1988 .

1980 Mathematics Subject Classification (1985 Revision). Primary 35N05; Secondary 58G05. 
Finally, $\S 3$ deals with the smooth integrals of a differential from $d Z$ whose singular locus has codimension $\geq 2$. We first recall the Whitney extension theorem for functions defined on compact subsets of $\mathbf{C}^{n}$. Then we give a "Taylor expansion" of the $C^{\infty}$-integrals, and prove an isomorphism theorem between the space $\mathscr{I}_{z}(\bar{U})$ of $C^{\infty}$-integrals of $d Z$ in $\bar{U}$ and $\mathscr{F}_{Z}^{\infty}(\bar{U})$, a space built from subspaces $\mathscr{E}_{H}^{\infty}(Z(\bar{U}))(j=1, \ldots, k)$ of the Whitney functions of class $C^{\infty}$ on the compact subsets $Z\left(\bar{U}_{j}\right)$ of $\mathbf{C}$. We conclude by showing how the pushforward of a $C^{k}$-integral of a real analytic hypoanalytic structure can be extended as a smooth function about $Z(0) \in \mathbf{C}$.

Acknowledgment. The author wishes to express his deepest gratitude to his teacher, Professor François Treves. He also thanks the referee for suggesting some changes to the first version of this paper.

\section{Definitions.}

1.1. Singular differential forms. In this section, we recall some properties of a singular real analytic differential form near $0 \in R^{n}$ and some results in the holomorphic category. Let

$$
\omega(x)=\sum_{i=1}^{n} a_{i}(x) d x_{i}, \quad x \in \mathbf{R}^{n},
$$

be a real analytic differential form in a neighborhood $U$ of $0 \in \mathbf{R}^{n}$, formally integrable, i.e.,

$$
d \omega \wedge \omega=0 .
$$

The singular locus of $\omega$ in $U$ is the real analytic variety

$$
\mathbf{S}_{U}(\omega)=\left\{x \in U ; a_{1}(x)=a_{2}(x)=\cdots=a_{n}(x)=0\right\} .
$$

If $\alpha=\operatorname{gcd}\left(a_{1}, \ldots, a_{n}\right)$ (the greatest common divisor of $\left.a_{1}, \ldots, a_{n}\right)$, then $\omega=\alpha \omega^{\prime}$, where $\omega^{\prime}$ is a real analytic differential form whose singular locus has codimension $\geq 2$. Since we are interested here by the integrals of $\omega$, then we can assume that the codimension of $\mathbf{S}_{U}(\omega)$ is $\geq 2$. In this case, it is proved in [7] that if $\eta$ is a $C^{\infty}$-differenital form such that $\eta \wedge \omega=0$, then

$$
\eta=g \omega,
$$

for some $C^{\infty}$-function $g$ near $0 \in \mathbf{R}^{n}$.

Denote by $\tilde{\omega}$ the complexified form of $\omega$, i.e.,

$$
\tilde{\omega}(\tilde{x})=\sum_{i=1}^{n} a_{i}(\tilde{x}) d \tilde{x}_{i}, \quad \tilde{x} \in \mathbf{C}^{n} .
$$

The differential form $\tilde{\omega}$ generates a foliation $\mathscr{F}_{\tilde{\omega}}$ outside its singular locus: The leaves of $\mathscr{F}_{\hat{\omega}}$ are the integral manifolds of $\tilde{\omega}$.

A germ at $0 \in \mathbf{C}^{n}$ of a holomorphic function $h$ is said to be an integral of $\tilde{\omega}$ if $\tilde{\omega} \wedge d h=0$, that is, the leaves of $\mathscr{F}_{\tilde{\omega}}$ are the irreducible components of the level sets of $h$. 
In [5], Mattei and R. Moussu show that $\tilde{\omega}$ has a holomorphic integral if and only if the closures, in a neighborhood of $0 \in \mathbf{C}^{n}$, of the leaves of $\mathscr{F}_{\tilde{\omega}}$ are analytic varieties and only a finite number of then adhere to 0 . They also prove a factorization result for the integrals: If $h$ and $f$ are integrals of $\tilde{\omega}$ and $h$ is not a power of a holomorphic function, then there is a germ of holomorphic function $l$ at $h(0) \in \mathbf{C}$ such that, $f=l \circ h$.

Also, Malgrange [4] gives sufficient conditions for the existences of strong integrals. More precisely, there are germs at $0 \in \mathbf{C}^{n}$ of holomorphic functions $g$ and $h$ with $g(0) \neq 0$ such that

$$
\tilde{\omega}=g d h,
$$

whenever one of the following conditions holds: (i) the codimension of $\mathbf{S}(\tilde{\omega})$ is $\geq 3$ or (ii) there are formal power series $g$ and $h$, with $g$ unit, that satisfy equation (1.13).

1.2. Integrals and fibers of a real analytic differential form. This section is devoted to the introduction of integrals and fibers above a point for a real analytic differential form. A special stratification that will be assumed throughout this paper is given at the end of this section. Denote by $C^{k}(U)$ the space of functions of class $C^{k}$ in $U$, where $k$ is either a nonnegative integer, or $k=\infty$, or $k=\bar{\omega}$ for real analytic functions, and denote by $C(U, \mathbf{C T} U)$ the space of smooth sections of the tangent bundle CT $U$. Let

$$
\mathbf{L}=\{L \in C(U, \mathbf{C T} U) ;\langle L, \omega\rangle=0\} .
$$

Then it follows from equation (1.11) that $\mathbf{L}$ is a Lie algebra, i.e., $\left[L, L^{\prime}\right] \in \mathbf{L}$ for all $L, L^{\prime} \in \mathbf{L}$. A function $f \in C^{k}(U)$ is said to be an integral of $\omega$ if

$$
L f=0 \text { for all } L \in \mathbf{L} \text {. }
$$

Note that when $k \geq 1$, equation (1.21) is equivalent to $d f \wedge \omega=0$.

From now on, we will assume that $\omega$ has a real analytic integral ( $\tilde{\omega}$ has a holomorphic integral) and we will refer to an integral $Z$ that is not a power of a real analytic function as a fundamental integral of $\omega$. As for the fibers of hypoanalytic structures, we define the fiber $F_{p}$ of $\omega$ above the point $p$ as $F_{p}=Z^{-1}(Z(p))$. We deduce the following proposition:

Proposition 1.21. Let $Z$ be a fundamental integral of the germ at $0 \in \mathbf{R}^{n}$ of a real analytic differential form $\omega$. Then there is a neighborhood $U$ of 0 on which

$$
\mathbf{S}_{U}(\omega) \subset \mathbf{S}_{U}(d Z) \subset F_{0} .
$$

Proof. Since $d Z \wedge \omega=0$ and $\operatorname{codim} \mathbf{S}_{U}(\omega) \geq 2$, then it follows from equation (1.12) that $d Z=g \omega$ for some real analytic function $g$. Thus, $\mathbf{S}_{U}(\omega) \subset$ $\mathbf{S}_{U}(d Z)$. Since the germ at 0 of $\mathbf{S}_{U}(d Z)$ is a subset of $F_{0}$, the proposition follows. 
From now on, $U$ will be as in Proposition 1.21 and will be written as

$$
U=\bigcup_{j=1}^{k} U_{j} \cup F_{0},
$$

where $U_{j}$ 's are the connected components of $U-F_{0}$.

\section{CONSTANCY ON THE FIBERS}

2.1. Statement and consequences of the main result. The main result of this section is

Theorem 2.11. There is a neighborhood $U$ of $0 \in \mathbf{R}^{n}$ such that if $f$ is a continuous integral of $\omega$ in $U$, then $f$ is constant on the fibers in the $U_{j}$ 's. More precisely,

$$
f\left(F_{x} \cap U_{j}\right)=f(x) \text { for all } x \in U_{j} .
$$

The proof of this theorem is the focus of $\S 2.2$. We give here some consequences of this result.

Theorem 2.12. Assume that the codimension of $F_{0} \geq 2$. Then there is a neighborhood $U$ of $0 \in \mathbf{R}^{n}$ such that if $f$ is a continuous integral of $\omega, f$ is constant on the fibers in $U$, i.e.,

$$
f\left(F_{x}\right)=f(x) \text { for all } x \in U \text {. }
$$

Proof. The codimension of $F_{0}$ being $\geq 2$, then $U-F_{0}$ is connected and Theorem 2.11 gives the result.

Corollary 2.11. Let $f$ be a continuous integral of $\omega$ in $\bar{U}$. Then there are continuous functions $\tilde{f}_{1}, \ldots, \tilde{f}_{k}$ defined respectively on $Z\left(\bar{U}_{1}\right), \ldots, Z\left(\bar{U}_{k}\right)$ such that

$$
\tilde{f}_{j}(Z(0))=\tilde{f}_{i}(Z(0)) \text { for all } i, j=1, \ldots, k
$$

and

$$
f(x)=\tilde{f}_{j} \circ Z(x) \text { for all } x \in U_{j} .
$$

Proof. Let $\sim$ be the equivalence relation "being the same fiber in $U_{j}$," that is,

$$
x \sim x^{\prime} \text { iff } x^{\prime} \in F_{x} \text { and } x, x^{\prime} \in \bar{U}_{j} .
$$

The quotient space $\bar{U}_{j} / \sim$ is then homeomorphic to $Z\left(\bar{U}_{j}\right)$. Since a continuous integral $f$ is constant on the fibers in $U_{j}$ (Theorem 2.11), there is $\tilde{f}_{j}$ as in (2.12). Also, since $f$ is continuous, (2.11) holds.

Corollary 2.12. Under the assumptions of Theorem 2.12, there is a continuous function $\tilde{f}$ on $Z(\bar{U})$ such that $f=\tilde{f} \circ Z$ in $\bar{U}$.

Remark 2.11. Polynomial approximation. It follows from Corollary 2.11 that there are $k$ sequences of polynomials $\left(P_{\nu}^{1}\right)_{\nu}, \ldots,\left(P_{\nu}^{k}\right)_{\nu}$ such that

$$
f=\lim _{\nu} P_{\nu}^{j} \circ Z \quad \text { in } U_{j} \text {. }
$$


Moreover, under the hypothesis of Theorem 2.12, there is a sequence of polynomials $\left(P_{\nu}\right)_{\nu}$ such that

$$
f=\lim _{\nu} P_{\nu} \circ Z \text { in } U .
$$

As for hypocomplex structures (see Treves [9]), we deduce

Corollary 2.13. Assume that $Z(U)$ is a neighborhood of $Z(0) \in \mathbf{C}$. Then every continuous integral of $\omega$ is real analytic.

Proof. From the fact that $Z(U)$ is a neighborhood of $Z(0) \in \mathbf{C}$, it follows that the codimension of $\{x \in U ; d Z(x) \wedge d \bar{Z}(x)=0\}$ is $\geq 1$ and that the codimension of $F_{0}$ is $\geq 2$. Now, if $f$ is a continuous integral of $\omega$, then $f=\tilde{f} \circ Z$ for some continuous function $\tilde{f}$ on $Z(U)$ (Corollary 2.12). Moreover, $\tilde{f}$ is holomorphic in $Z(U-\{x ; d Z(x) \wedge d \bar{Z}(x)=0\}) \quad(Z$ is a complex structure there). Therefore, $\tilde{f}$ is holomorphic in a neighborhood of $Z(0)$ and so $f$ is real analytic.

\section{Examples.}

1. The continuous integrals of $x d x+i y d y$, or equivalently the continuous solutions of

$$
\left(-i y \frac{\partial}{\partial x}+x \frac{\partial}{\partial y}\right) f(x, y)=0
$$

satisfy $f\left(x_{0}, y_{0}\right)=f\left(-x_{0}, y_{0}\right)=f\left(-x_{0},-y_{0}\right)=f\left(x_{0},-y_{0}\right)$ for $\left(x_{0}, y_{0}\right) \in$ $\mathbf{R}^{2}$. That is, we have constancy on the fibers because the codimension of $F_{(0,0)}=\{(0,0)\}$ is 2 .

2. A fundamental integral of $\omega=\left(2 x^{2}+i y^{2}\right) d x+i x y d y$ in $\mathbf{R}^{2}$ is $Z=$ $x^{4}+i x^{2} y^{2}$. The stratification here is $\{x>0\} \cup\{x<0\} \cup\{x=0\}$. The integrals are constant on the fibers in $\{x>0\}$ and in $\{x<0\}$ separately, as for the following $C^{\infty}$-integral:

$$
f(x, y)=\left\{\begin{array}{l}
\exp \frac{-1}{x^{4}+i x^{2} y^{2}} \quad \text { if } x \geq 0 \\
0 \text { if } x \leq 0
\end{array}\right.
$$

3. All the integrals of $\omega=x^{2} d x+i y^{2} d y$ are real analytic. Indeed, $\omega$ admits $Z=x^{3}+i y^{3}$ as a fundamental integral, and the image via $Z$ of neighborhood of $0 \in \mathbf{R}^{2}$ is a neighborhood of $0 \in \mathbf{C}$.

2.2. Proof of Theorem 2.11. We distinguish two cases:

Case 1. $d Z \wedge d \bar{Z} \equiv 0$. We can assume in this case that $\omega$ is real-valued. Indeed, we have $d \bar{Z} \wedge \omega=0$, that is, $\bar{Z}$ is an integral of $\omega$ and so are the real-valued functions $Z+\bar{Z}$ and $i(Z-\bar{Z})$. The proof of this case is contained in [6].

Case 2. $d Z \wedge d \bar{Z} \neq 0$. Let

$$
\Sigma=\{x \in U ; d Z(x) \wedge d \bar{Z}(x)=0\} .
$$


The germ at $0 \in \mathbf{R}^{n}$ of $\Sigma$ is an analytic variety of dimension $\leq n-1$. For $j$ fixed in $\{1, \ldots, k\}$, let $\Sigma_{j}=\Sigma \cap U_{j}$. Since the structure generated by $Z$ is hypoanalytic in $U_{j}$ (i.e., $d Z \neq 0$ in $U_{j}$ ), we distinguish two kinds of points on $\Sigma_{j}$ : the points $p$ where $Z$ is hypocomplex (the image via $Z$ of an open neighborhood of $p$ is an open neighborhood of $Z(p)$ in $\mathbf{C})$, and the others. Let us write $\Sigma_{j}=H_{j} \cap N_{j}$, where $H_{j}$ is the set of points of $\Sigma_{j}$ where $Z$ is hypocomplex and $N_{j}=\Sigma_{j}-H_{j}$. We write

$$
U_{j}-N_{j}=\bigcup_{l=1}^{k_{j}} U_{j}^{l},
$$

where the $U_{j}^{l}$,s are the connected components of $U_{j}-N_{j}$.

Lemma 2.21. There is a neighborhood $U$ of 0 on which $F_{x} \cap U_{j}^{l}$ is connected for every $x \in U_{j}^{l}, l \leq k_{j}$ and $j \leq k$.

Proof. We first prove the lemma for $n=2$. We write

$$
U_{j}^{l}-H_{j}=\bigcup_{m=1}^{r_{l}} U_{j}^{l, m}
$$

where $U_{j}^{l, m}$, s are the connected components of $U_{j}^{l}-H_{j}$. It is enough to prove that one can find $U$ such that $F_{x} \cap U_{j}^{l, m}$ is connected and that $Z\left(U_{j}^{l, m}\right) \cap$ $Z\left(U_{j}^{l, m^{\prime}}\right)=\varnothing$ if $m \neq m^{\prime}$.

Since $Z$ is a complex structure in $U_{j}^{l, m}$, there is $\varepsilon>0$ such that

$$
Z: U_{j}^{l, m} \cap D(0, \varepsilon) \mapsto Z\left(U_{j}^{l, m} \cap D(0, \varepsilon)\right) \subset C,
$$

where $D(0, \varepsilon)$ denotes the disk with radius $\varepsilon$ centered at 0 , is a diffeomorphism (otherwise, there would be analytic curves $C_{1}, C_{2}$ in $U_{j}^{l, m}$ and passing through 0 such that $Z\left(C_{1}\right)=Z\left(C_{2}\right)$ and then $d Z \wedge d \bar{z}$ would vanish along a third curve between $C_{1}$ and $\left.C_{2}\right)$. We take $U_{j}^{l, m} \cap D(0, \varepsilon)$ as the new $U_{j}^{l, m}$.

Next, by contradiction, assume that there are distinct $m$ and $m^{\prime}$ such that the germ at $Z(0)$ of $Z\left(U_{j}^{l, m}\right) \cap Z\left(U_{j}^{l, m^{\prime}}\right)$ is not empty. Then, we could choose analytic curves $C^{m}$ and $C^{m^{\prime}}$, respectively, in $U_{j}^{l, m}$ and $U_{j}^{l, m^{\prime}}$ and passing through 0 such that

$$
Z\left(C^{m}\right)=Z\left(C^{m^{\prime}}\right) .
$$

For $\varepsilon>0$, consider the analytic set $A_{\varepsilon} \in U_{j}^{l}$ that is bounded by $C^{m}, C^{m^{\prime}}$, and the circle with radius $\varepsilon$ centered at 0 . Then it would follow from (2.21) that the boundary of the subanalytic set $Z\left(\bar{A}_{\varepsilon}\right)$ would not be $Z\left(\partial A_{\varepsilon}\right)$, that is, $Z$ would not be hypocomplex in $A_{\varepsilon} \subset U_{j}^{l}$. We have a contradiction, and the lemma is proved in this case.

For $n>2$, we consider a real analytic embedding $\phi:\left(\mathbf{R}^{2}, 0\right) \mapsto\left(\mathbf{R}^{2}, Z(0)\right)$ such that the image via $\phi$ of a small neighborhood $V$ of $0 \in \mathbf{R}^{2}$ is generically transveral to the fibers of $Z$ (i.e., $d(Z \circ \phi)$ is not identically 0 ). The open set 
$\bigcup_{x \in \phi(V)} C_{x}$, where $V$ is the open neighborhood of $0 \in \mathbf{R}^{2}$ constructed above for the function $Z \circ \phi$ and where $C_{x}$ is the connected component through $x$ of $F_{x}$, satisfies the lemma.

To continue the proof of the theorem, consider $x_{0} \in U_{j}$ and let

$$
F_{x_{0}} \cap U_{j}=\Gamma_{j}^{l_{1}} \cup \cdots \cup \Gamma_{j}^{l_{\nu}},
$$

where $\Gamma_{j}^{l_{p}}$ denotes the connected component of $F_{x_{0}}$ in $U_{j}^{l_{p}}$. Let us show that if $f$ is a continuous integral of $\omega$, then $f\left(\Gamma_{j}^{l_{1}}\right)=\cdots=f\left(\Gamma_{j}^{l_{\nu}}\right)$. For this, we distinguish two cases:

Case a. $U_{j}^{l_{1}}$ and $U_{j}^{l_{2}}$ are adjacent. Let $C_{j}$ be the common part of their boundary and let $\gamma_{1}$ be a simple curve in $U_{j}^{l_{1}}$ that joins a point in $\Gamma_{j}^{l_{1}}$ with a point $\alpha \in C_{j}$, and such that $Z\left(\gamma_{1}\right)$ is a simple curve in $Z\left(U_{j}^{l_{1}}\right)$. Let $\gamma_{2}$ be the reflection of $\gamma_{1}$ in $U_{j}^{l_{2}}$ that starts from $\alpha$ (by reflection, we mean that $\left.Z\left(\gamma_{1}\right)=Z\left(\gamma_{2}\right)\right)$. Since the structure defined by $Z$ is hypoanalytic in $U_{j}$, for every $a \in \gamma_{1}$ there is an open neighborhood $O_{a}$ of $a$ and a continuous function $h_{a}$ defined on the closure of $Z\left(O_{a}\right)$ and holomorphic in its interior such that $f=h_{a} \circ Z$ in $O_{a}$ (see [2]). By analytic continuation, we deduce the existence of an open neighborhood $O_{1}$ of $\gamma_{1}$ such that $Z\left(O_{1}\right)$ is simply connected and of a continuous function $G_{1}$ defined on the closure of $Z\left(O_{1}\right)$, holomorphic in the interior of $Z\left(O_{1}\right)$ such that $f=G_{1} \circ Z$ in $O_{1}$. We repeat the argument with $\gamma_{2}$ to find $O_{2}$ and $G_{2}$, such that $f=G_{2} \circ Z$ in $O_{2}$. Now, $\tau=Z\left(O_{1} \cap O_{2} \cap C_{j}\right)$ is a real analytic curve and $G_{1}=G_{2}$ on $\tau$. Then, by analytic continuation we obtain $G_{1}=G_{2}$ on the closure of $Z\left(O_{1}\right) \cap Z\left(O_{2}\right)$. The theorem is proved in this situation.

Case b. $U_{j}^{l_{1}}$ and $U_{j}^{l_{2}}$ are not adjacent. We can choose a sequence $U_{j}^{l_{1}}, U_{j}^{p_{1}}$, $\ldots, U_{j}^{p_{k}}, U_{j}^{l_{2}}$ such that the couples $\left(U_{j}^{l_{1}}, U_{j}^{p_{1}}\right), \ldots,\left(U_{j}^{p_{i}} U_{j}^{p_{i+1}}\right), \ldots,\left(U_{j}^{p_{k}}, U_{j}^{l_{2}}\right)$ are adjacent. Denote by $C_{1}$ the common boundary of $\left(U_{j}^{l_{1}}, U_{j}^{p_{1}}\right)$, by $C_{i}$ the common boundary of $\left(U_{j}^{p_{i-1}}, U_{j}^{p_{i}}\right)$, and by $C_{2}$ that of $\left(U_{j}^{p_{k}}, U_{j}^{l_{2}}\right)$. Let $\gamma_{1}$ be a simple curve in $U_{j}^{l_{1}}$ that joins a point in $\Gamma_{j}^{l_{1}}$ to a point $\alpha_{1} \in C_{1}$ such that $Z\left(\gamma_{1}\right)$ is simple. Let $\gamma^{1}$ be the reflection in $U_{j}^{p_{1}}$ of

$$
\gamma_{1} \cap Z^{-1}\left(Z\left(\gamma_{1}\right)\right) \cap Z^{-1}\left(Z\left(U_{j}^{p_{1}}\right)\right)
$$

that starts from $\alpha_{1} \in C_{1}$ and arrives to a point $\alpha^{1} \in C^{1}$. We define by induction $\gamma^{i+1}$ as the reflection in $U_{j}^{p_{i+1}}$ of

$$
\gamma_{1} \cap Z^{-1}\left(Z\left(\gamma_{1}\right)\right) \cap Z^{-1}\left(Z\left(U_{j}^{p_{i+1}}\right)\right)
$$

that starts from $\alpha^{i} \in C^{i}$ and arrives to $\alpha^{i+1} \in C^{i+1}$. At the end, we reach a point in $\Gamma_{j}^{l_{2}} \subset U_{j}^{l_{2}}$ by a curve $\gamma_{2}$. We repeat the argument of Case a for 
each couple $\left(\gamma^{i}, \gamma^{i+1}\right)$ to find that $f\left(\Gamma_{j}^{l_{1}}\right)=f\left(\Gamma_{j}^{l_{2}}\right)$. The theorem is therefore proved.

\section{SMOOTh INTEGRALS AS WhitNey FUNCTIONS}

3.1. Whitney functions. We recall in this section the notion of Whitney functions and the extension theorem. Let $K$ be a compact subset of $C^{n}$ and $J^{r}(K)$ be the space of jets of order $r$ of continuous functions on $K$, that is,

$$
J^{r}(K)=\left\{F=\left(F^{(\alpha, \beta)}\right)_{|\alpha|+|\beta| \leq r} ; \alpha, \beta \in \mathbf{N}^{n} \text { and } F^{(\alpha, \beta)} \in \mathbf{C}^{0}(K, \mathbf{C})\right\} .
$$

The operators

$$
\begin{aligned}
& J^{r}: C^{r}\left(\mathbf{C}^{n}\right) \mapsto J^{r}(K) \\
& f \mapsto J^{r}(f)=\left(\frac{\partial^{|\alpha|+|\beta|}}{\partial z^{\alpha} \partial \bar{z}^{\beta}} f\right)_{|\alpha|+|\beta| \leq r}
\end{aligned}
$$

and

$$
\begin{aligned}
& D^{(k, l)}: J^{r}(K) \mapsto J^{r-(|k|+|l|)}(K), \\
& F \mapsto D^{(k, l)} F=\left(F^{(\alpha, \beta)+(k, l)}\right)_{|\alpha|+|\beta| \leq r-(|k|-|l|)},
\end{aligned}
$$

where $k, l \in \mathbf{N}^{n}$ and $|k|+|l| \leq r$, satisfy

$$
D^{(k, l)} \circ J^{r}=J^{r-(|k|+|l|)} \circ \frac{\partial^{|k|+|l|}}{\partial z^{k} \partial \bar{z}^{l}} .
$$

If $p \in K$ and $f \in J^{r}(K)$, then the $r$ th "Taylor polynomial" of $F$ at $p$ is defined as

$$
T_{p}^{r} F(z)=\sum_{|\alpha|+|\beta| \leq r} \frac{1}{\alpha ! \beta !} F^{(\alpha, \beta)}(p)(z-p)^{\alpha}(\bar{z}-\bar{p})^{\beta}
$$

and the "remaining" as

$$
R_{p}^{r} F=F-J^{r}\left(T_{p}^{r} F\right)
$$

Definition 3.11 [8]. A jet $F \in J^{r}(K)$ is called a Whitney function of class $C^{r}$ on $K$ if

$$
\left(R_{p}^{r} F\right)^{(\alpha, \beta)}(p)=o\left(|p-q|^{r-(|\alpha|+|\beta|)}\right),
$$

for all $\alpha, \beta \in \mathbf{N}^{n}$ and $p, q \in K$.

Denote by $\mathscr{E}^{r}(K)$ the space of Whitney functions of class $C^{r}$ on $K$ and consider the map

$$
\begin{aligned}
\Pi_{r, r+1}: J^{r+1}(K) & \mapsto J^{r}(K), \\
\left(F^{(\alpha, \beta)}\right)_{|\alpha|+|\beta| \leq r+1} & \mapsto\left(F^{(\alpha, \beta)}\right)_{|\alpha|+|\beta| \leq r} .
\end{aligned}
$$

Then clearly we have $\Pi_{r, r+1}\left(\mathscr{E}^{r+1}(K)\right) \subset \mathscr{E}^{r}(K)$. The projective limit $\mathscr{E}^{\infty}(K)$ of $\mathscr{E}^{r}(K)$ (resp. $J^{\infty}(K)$ of $J^{r}(K)$ ) is called the space of Whitney functions $C^{\infty}$ (resp. the space of jets of infinite order) on $K$. Recall the Whitney extension theorem: 
Theorem (Whitney [10]). For every $r(r \leq \infty)$ there is a linear map $W: \mathscr{E}^{r}(K)$ $\mapsto C^{r}\left(C^{n}\right)$ such that for every $F \in \mathscr{E}^{r}(K)$ and for every $p \in K$ the following holds:

$$
\frac{\partial^{|\alpha|+|\beta|}}{\partial z^{\alpha} \partial \bar{z}^{\beta}}(W F)(p)=F^{(\alpha, \beta)}(p) \quad \text { if }|\alpha|+|\beta| \leq r .
$$

Moreover, the restriction of $W F$ to $C^{n}-K$ is a $C^{\infty}$ function.

The proof of this theorem can also be found in Tougeron [8].

3.2. A Taylor expansion of $C^{\infty}$-integrals. Let $U$ be an open neighborhood of $0 \in \mathbf{R}^{N}$ and $Z$ be a complex-valued real analytic function in $U$ such that the codimension of $\mathbf{S}_{U}(d Z)$ is $\geq 2$. Consider the spaces

$$
\mathscr{I}_{Z}(U)=\left\{f \in C^{\infty}(U) ; d f \wedge d Z=0\right\}
$$

(the space of $C^{\infty}$-integrals of $d Z$ in $U$ ) and $\mathscr{I}_{Z}(\bar{U})=\mathscr{I}_{Z}(u) \cap C^{\infty}(\bar{U})$. It follows from (1.12) that if $f \in \mathscr{I}_{Z}(U)$, then there is $\mathscr{D}(f) \in C^{\infty}(U)$ such that

$$
d f=\mathscr{D}(f) d Z \text {. }
$$

Lemma 3.21. $\mathscr{D}(f) \in \mathscr{I}_{Z}(U)$.

Proof. The structure generated by $d Z$ is hypoanalytic in $U-\mathbf{S}_{U}(d Z)$. Thus, it follows from the general theory of hypoanalytic structures (see Treves [9]) that $\mathscr{D}(f)$ is an integral there. Since $U-\mathbf{S}_{U}(d Z)$ is dense in $U$ and $\mathscr{D}(f)$ is $C^{\infty}$, the conclusion follows.

The operator $\mathscr{D}: \mathscr{I}_{Z}(U) \mapsto \mathscr{I}_{Z}(U)$ is "the derivative with respect to $Z$ " and if $d Z \neq 0$, then $\mathscr{D}$ is the vector field $M$ introduced in [9]. Let

$$
\mathscr{D}^{n}=\mathscr{D} \circ \cdots \circ \mathscr{D}, \quad n \text {-times, }
$$

and define the $r$ th "Taylor polynomial" of $f$ at $p$ as

$$
T_{p}^{r} f(x)=\sum_{j=1}^{r} \frac{1}{j !} \mathscr{D}^{j} f(p)(Z(x)-Z(p))^{j} .
$$

Denote by $\Gamma_{(Z(x), Z(p))}$ a curve in $Z(U)$ that joins $Z(x)$ with $Z(p)$ and by $l\left(\Gamma_{(Z(x), Z(p))}\right)$ is length. Then

Proposition 3.21. If $f \in \mathscr{J}_{Z}(\bar{U})$, then

$$
f(x)-T_{p}^{r} f(x)=o\left(l\left(\Gamma_{(Z(x), Z(p))}\right)^{r}\right) .
$$

Proof. It follows from equation (3.21) that

$$
f(x)-T_{p}^{r} f(x)=\int_{\gamma(x, p)} \mathscr{D} f\left(y_{1}\right) d Z\left(y_{1}\right),
$$

where $\gamma(x, p)$ is any rectifiable curve in $U$ that joins $x$ to $p$. Since $\mathscr{D} f \in$ $\mathscr{I}_{Z}(U)$, then we can apply equation (3.22) to it and obtain

$$
D f\left(y_{1}\right)=\int_{\gamma\left(y_{1}, p\right)} \mathscr{D}^{2}\left(y_{2}\right) d Z\left(y_{2}\right)+\mathscr{D} f(p) .
$$


Combine equations (3.22) and (3.23) to get

$$
f(x)-T_{p}^{1} f(x)=\int_{\gamma\left(x, y_{1}\right)} \int_{\gamma\left(y_{1}, p\right)} \mathscr{D}^{2} f\left(y_{2}\right) d Z\left(y_{2}\right) \wedge d Z\left(y_{1}\right) .
$$

We continue this process $r$ times to find

$$
f(x)-T_{p}^{r} f(x)=\int_{\gamma\left(x, y_{1}\right)} \cdots \int_{\left.\gamma\left(y_{r}, p\right)\right)} \mathscr{D}^{r+1} f\left(y_{r+1}\right) d Z\left(y_{r+1}\right) \wedge \cdots \wedge d Z\left(y_{1}\right) .
$$

Let $c=\max \left\{\mathscr{D}^{r+1} f(y) ; y \in \bar{U}\right\}$. Then

$$
\left|f(x)-T_{p}^{r} f(x)\right| \leq \operatorname{cl}\left(\Gamma_{(Z(x), Z(p))}\right)^{r+1}
$$

and the proposition is proved.

3.3. An isomorphism. We prove in this section that the space of $C^{\infty}$-integrals $\mathscr{I}_{Z}(\bar{U})$ is isomorphic to a space $\mathscr{F}_{Z}^{\infty}(\bar{U})$, built from subspaces $\mathscr{E}_{H}^{\infty}\left(Z\left(\bar{U}_{j}\right)\right)$ of the spaces of Whitney functions $\mathscr{E}^{\infty}\left(Z\left(\bar{U}_{j}\right)\right)$, where $U=\bigcup_{j=1}^{k} U_{j} \cup F_{0}$ is the stratification of $\S 1.2$. For this purpose, we consider the following spaces:

$$
\begin{gathered}
J_{H}^{r}(K)=\left\{F \in J^{r}(K) ; F^{(\alpha, \beta)}=0 \text { if } \beta \neq 0\right\}, \\
L_{Z}^{r}(\bar{U})=\left\{\left(F_{1}, \ldots, F_{k}\right) \in J_{H}^{r}\left(Z\left(\bar{U}_{1}\right)\right) \times \cdots \times J_{H}^{r}\left(Z\left(\bar{U}_{k}\right)\right) ; F_{1}(0)=\cdots=F_{k}(0)\right\}, \\
\mathscr{E}_{H}^{r}(K)=\mathscr{E}^{r}(K) \cap J_{H}^{r}(K),
\end{gathered}
$$

and

$$
\mathscr{F}_{Z}^{r}(\bar{U})=\mathscr{E}_{H}^{r}\left(Z\left(\bar{U}_{1}\right)\right) \times \cdots \times \mathscr{E}_{H}^{r}\left(Z\left(\bar{U}_{k}\right)\right) \cap L_{Z}^{r}(\bar{U}) .
$$

We denote by $\mathscr{F}_{Z}^{\infty}(\bar{U})$ (resp. $L_{Z}^{\infty}(\bar{U})$ ) the projective limit of $\mathscr{F}_{Z}^{r}(\bar{U})$ (resp. $\left.L_{Z}^{r}(\bar{U})\right)$. We have the following theorem:

Theorem 3.31. $\mathscr{I}_{Z}(\bar{U})$ is isomorphic to $\mathscr{F}_{Z}^{\infty}(\bar{U})$.

Proof. Let $\mathscr{B}_{r}: \mathscr{I}_{Z}(\bar{U}) \mapsto L_{Z}^{r}(\bar{U})$ be defined by

$$
\mathscr{B}_{r}(f)=\left(\widetilde{\mathscr{D}^{l}} f\right)_{1}, \ldots,\left(\widetilde{\mathscr{D}^{l}} f_{k}\right)_{l \leq r},
$$

where $\tilde{h}_{j}$ denotes the pushforward of the integral $h$ of $d Z$ in $U_{j}$ (see Corollary 2.11). And let

$$
\mathscr{B}=\lim \mathscr{B}_{r}: \mathscr{I}_{Z}(\bar{U}) \mapsto L_{Z}^{\infty}(\bar{U})
$$

Note that since $\Pi_{r, r+1} \circ \mathscr{B}_{r+1}=\mathscr{B}_{r}, \mathscr{B}$ is well defined. In order to continue, we need a lemma whose proof is given in the next section.

Lemma 3.31. $\mathscr{B}\left(\mathscr{I}_{Z}(\bar{U})\right) \subset \mathscr{F}_{Z}^{\infty}(\bar{U})$.

Now, define the operator $\mathscr{P}: \mathscr{F}_{Z}^{\infty}(\bar{U}) \mapsto \mathscr{I}_{Z}(\bar{U})$ by

$$
\mathscr{P}\left(F_{1}, \ldots, F_{k}\right)(x)=\left(W F_{j}\right) \circ Z(x) \text { if } x \in U_{j},
$$

where $W$ is the Whitney extension operator. It follows from the fact $F_{1}(0)=$ $\cdots=F_{k}(0)$ that $\mathscr{P}(F)$ is $C^{\infty}$ and from the fact $(\partial / \partial \bar{Z})\left(W F_{j}\right)(Z(x))=0$ that

$$
d \mathscr{P}(F) \wedge d Z=0
$$


That is, $\mathscr{P}$ is well defined. Also

$$
\mathscr{D}^{l}(\mathscr{P}(F))(x)=\frac{\partial^{l}}{\partial Z^{l}}\left(W F_{j}\right)(Z(X)) \quad \text { if } x \in U_{j} .
$$

Therefore $\mathscr{P}^{-1}=\mathscr{B}$ and the theorem is proved.

Proof of lemma. By letting $r$ go to $\infty$, it is enough to show $\mathscr{B}_{r} f \in \mathscr{F}_{Z}^{r}(\bar{U})$ for every $f \in \mathscr{I}_{Z}(\bar{U})$, i.e., we have to prove

$$
\left(R_{a}^{r}\left(\mathscr{B}_{r} f\right)_{j}\right)^{l}(b)=o\left(|b-a|^{r-1}\right), \quad j=1, \ldots, k,
$$

where $\mathscr{B}_{r} f\left(\left(\mathscr{B}_{r} f\right)_{1}, \ldots,\left(\mathscr{B}_{r} f\right)_{k}\right)$ and $a, b$ are arbitrary points in $Z\left(\bar{U}_{j}\right)$. For this purpose, we use the following property of subanalytic sets to relate the geodesic distance to the Euclidian distance (see Hardt [3])

(*) If $\mathscr{A}$ is a connected compact subanalytic set in $\mathbf{C}^{m}$, then there is a rational number $\sigma \quad(0<\sigma \leq 1)$ and a positive constant $c$ so that any two points $a, b \in \mathscr{A}$ can be joined by a simple arc of length $\leq c|a-b|^{\sigma}$.

Let $\sigma_{j}, c_{j}$ be the constants of property $(*)$ for the subanalytic set $Z\left(\bar{U}_{j}\right)$ and let $\sigma=\min \left\{\sigma_{j} ; j=1, \ldots, k\right\}$. Choose $n \in \mathbf{N}$ such that $n \sigma \geq r+1$. Then it follows from property $(*)$ and Proposition 3.21 that

$$
f(x)-T_{p}^{r} f(x)=\sum_{\alpha=r+1}^{n} \frac{1}{\alpha !} \mathscr{D}^{\alpha} f(p)(Z(x)-Z(p))^{\alpha}+o\left(|Z(x)-Z(p)|^{n \sigma}\right) .
$$

So, with the choice of $n$, we have

$$
f(x)-T_{p}^{r} f(x)=o\left(|Z(x)-Z(p)|^{r+1}\right) .
$$

Finally, let $a_{j} \in Z^{-1}(a) \cap \bar{U}_{j}$ and $b \in Z^{-1}\left(\bar{U}_{j}\right)$, then

$$
\begin{aligned}
\left(R_{a}^{r}\left(\mathscr{B}_{r} f\right)_{j}\right)^{l}(b) & =\left(\mathscr{D}^{l}\left(\widetilde{f-T}_{a j}^{r} f\right)\right)_{j}(b) \\
& =\mathscr{D}^{l}\left(f-T_{a j}^{r} f\right)\left(b_{j}\right) \\
& =o\left(|b-a|^{r-1}\right) .
\end{aligned}
$$

Equation (3.31) is proved and so is the lemma.

As a consequence of this result and Theorem 3.22, we have

Theorem 3.32. Assume that the codimension of $F_{0}$ is $\geq 2$. Then $\mathscr{I}_{Z}(\bar{U})$ is isomorphic to $\mathscr{E}_{H}^{\infty}(Z(\bar{U}))$.

3.4. The real analytic hypoanalytic case. In this final section, we go back to the starting point of this work: the hypoanalytic structures. We consider a real analytic hypoanalytic structure generated by a function $Z(x)$ (with $d Z(0) \neq$ 0 ) and prove an extension theorem for the pushforward of its integrals (or solutions). 
Let $Z(x)$ be the germ at $0 \in \mathbf{R}^{n}$ of a real analytic function such that $d Z(0) \neq$ 0 and let $U$ be a neighborhood of 0 on which all the continuous integrals of $d Z$ are constant on the fibers. The subset $Z(\bar{U})$ of $\mathbf{C}$ being subanalytic, there are constants $c$ and $\sigma$ with $0<\sigma \leq 1$ such that any two points $a, b \in Z(\bar{U})$ can be joined by a simple rectifiable are in $Z(\bar{U})$ whose length is $\leq c|a-b|^{\sigma}$.

We will denote by $S^{k}(\bar{U})$ the space of $C^{k}$-integrals of $d Z$ in $\bar{U}$ and by $M$ the "derivation" with respect to $Z$, i.e., if $f \in S^{k}(\bar{U})$, then $d f(x)=$ $(M f)(x) d Z(x)$ where $M f \in S^{k-1}(\bar{U})$ (see Treves [9]). As in the previous section, we define

$$
\begin{aligned}
& \mathscr{B}_{k}: S^{k}(\bar{U}) \mapsto J_{H}^{k}(Z(\bar{U})), \\
& f \mapsto\left(f, M F, \ldots, M^{k-1} f\right) .
\end{aligned}
$$

The notation $[r]$ will be used for the greatest integer less than or equal to $r$. Then the proof of Theorem 3.31 can be carried out to show the following:

Theorem 3.41. $\mathscr{B}_{k}\left(S^{[k / \sigma]+1}(\bar{U})\right) \subset \mathscr{E}_{H}^{k}(Z(\bar{U}))$. That is, the pushforward of a $C^{[k / \sigma]+1}$-integral can be extended as a $C^{k}$-function in a neighborhood of $Z(0) \in$ C.

\section{REFERENCES}

1. M. S. Baouendi and F. Treves, $A$ property of functions and distributions annihilated by a locally integral system of vector fields, Ann. of Math. 113 (1981), 341-421.

2. _ A local constancy principle for the solutions of certain overdetermined systems of firstorder linear PED, Volume in honour of L. Schwartz, Advances in Math., North-Holland, 1981.

3. R. Hardt, Some analytic bounds for subanalytic sets, Differential Geometric Control Theory (Houghton, Mich., 1982), Progress in Math., vol. 27, Birkhäuser, Boston, Mass., 1983, pp. 259-267.

4. B. Malgrange, Frobenius avec singularités 1. Codimension un, Publ. Math. I.H.E.S. 46 (1976), 163-173.

5. J. F. Mattei and R. Moussu, Holonomie et intégrales premières, Ann. Sci. Ėcole Norm. Sup. (4) 13 (1980), 469-523.

6. R. Moussu, Sur lexistence d'intégrales premières pour un germe de forme de Pfaff, Ann. Inst. Fourier (Grenoble) 26 (1976), 171-220.

7. K. Saito, On a generalization of the de Rham lemma, Ann. Inst. Fourier (Grenoble) 26 (1976), 165-170.

8. J. C. Tougeron, Idéaux de fonctions différentiables, Springer-Verlag, Berlin, Heildelberg and New York, 1972.

9. F. Treves, Approximation and representations of functions and distributions annihilated by a system of vector fields, Ecole Polytechnique Centre de Mathématique, 1981.

10. H. Whitney, Analytic extensions of differentiable functions defined on closed sets, Trans. Amer. Math. Soc. 36 (1934), 63-89.

Department of Mathematics, Florida International University, University Park, MIAMI, FLORIDA 33199 